\title{
Multimedia Event Detection Using Event-Driven Multiple Instance Learning
}

\author{
Sang Phan ${ }^{\star \dagger}$, Duy-Dinh Le ${ }^{\dagger \S}$, Shin'ichi Satoh ${ }^{\dagger}$ \\ `The Graduate University for Advanced Studies (SOKENDAI), Japan \\ ${ }^{\dagger}$ National Institute of Informatics, Japan \\ $\S$ Multimedia Communications Lab, University of Information Technology, Vietnam \\ \{plsang,ledduy,satoh\}@nii.ac.jp
}

\begin{abstract}
A complex event can be recognized by observing necessary evidences. In the real world scenarios, this is a difficult task because the evidences can happen anywhere in a video. A straightforward solution is to decompose the video into several segments and search for the evidences in each segment. This approach is based on the assumption that segment annotation can be assigned from its video label. However, this is a weak assumption because the importance of each segment is not considered. On the other hand, the importance of a segment to an event can be obtained by matching its detected concepts against the evidential description of that event. Leveraging this prior knowledge, we propose a new method, Event-driven Multiple Instance Learning (EDMIL), to learn the key evidences for event detection. We treat each segment as an instance and quantize the instance-event similarity into different levels of relatedness. Then the instance label is learned by jointly optimizing the instance classifier and its related level. The significant performance improvement on the TRECVID Multimedia Event Detection (MED) 2012 dataset proves the effectiveness of our approach.
\end{abstract}

\section{Categories and Subject Descriptors}

I.2.10 [Artificial Intelligence]: Vision and Scene Understanding - Video analysis

\section{General Terms}

Algorithms, Experimentation, Measurement

\section{Keywords}

Event Detection, Event-driven, Multiple Instance Learning

\section{INTRODUCTION}

The problem of recognizing complex event in videos has become a popular research topic due to the explosive growth of video data. A complex event can involve several actions

(C) 2015 Association for Computing Machinery. ACM acknowledges that this contribution was authored or co-authored by an employee, contractor or affiliate of a national government. As such, the Government retains a nonexclusive, royalty-free right to publish or reproduce this article, or to allow others to do so, for Government purposes only.

MM'15, October 26-30, 2015, Brisbane, Australia.

(C) 2015 ACM. ISBN 978-1-4503-3459-4/15/10 ...\$15.00.

DOI: http://dx.doi.org/10.1145/2733373.2806330.

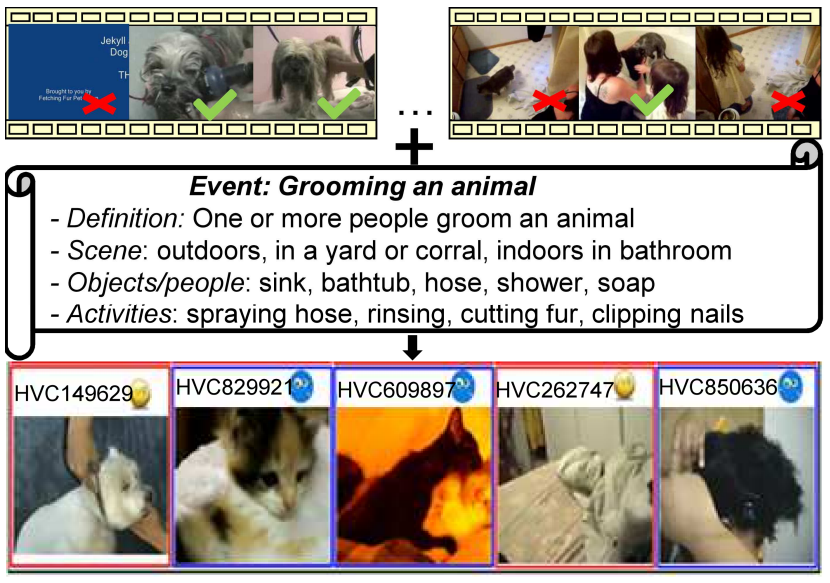

Figure 1: Event "Grooming an animal" in the TRECVID MED 2012 dataset. The event kit includes example videos and an event description which provides valuable cues to detect that event.

or activities and happens in some particular settings. Therefore, recognizing complex event is more challenging than single action recognition. However, most complex detection systems are still based on the techniques that was developed for action recognition $[6,9]$. These methods basically extract and aggregate local feature descriptors from the whole video to create a unique video representation. This strategy might be not effective for complex event detection because it treats different parts of the video equally. Therefore, it neutralizes the important local information of an event.

In practice, human can recognize a complex event by spotting several evidences in video [2]. This paper also demonstrated that better performance can be obtained by leveraging positive and negative visual cues selected by humans. Therefore, it is important to automatically detect key evidences for event detection. Several researchers have been working on this direction. Tang et al. [7] split the video into segments and models key segments and its duration as latent variables. Vahdat et al. [8] focus on intra-class variation by localizing only the most salient evidence using latent SVM. Lai et al. [5] detect salient instances in video based on a variant multiple instance learning. In another work [4], they represent static and dynamic instances as sparse features and adopt a learning-to-rank strategy to detect key evidence. In general, these approaches are based on the assumption that segment annotation can be obtained from its 
video label. However, this is a weak assumption because the importance of each segment is not taken into account.

On the other hand, the importance of a segment to an event can be obtained by matching its concept-based representation against the evidential description of that event. Some works have been using the event description for zeroshot event detection such as in $[3,10]$. To the best of our knowledge, no work has taken into account this information for detecting key evidence in videos. However, the evidential description of an event provides valuable information to detect that event. Example of an event description (excerpted) is shown in Fig. 1.

Motivated by this observation, we propose a new method, Event-driven Multiple Instance Learning (EDMIL), to learn key evidences for complex event detection. We treat each segment as an instance and model it in a multiple instance learning framework [1], where each video is a "bag". The instance-event similarity is quantized into different levels of relatedness. Intuitively, the most (ir)relevant instances should have higher (dis)similarities. Therefore, we propose to learn the instance labels by jointly optimize the instance classifier and its related level. We evaluate our proposed method on the large scale TRECVID MED 2012 dataset. Comparing to other instance-based learning methods such as $[1,5]$, our method achieves a superior performance.

The remaining of this paper is organized as follows. In the next section, we present the method to calculate the instance-event similarity. Our proposed solution is introduced in Section 3. The experiments and results are shown in Section 4. Finally, Section 5 concludes the paper.

\section{INSTANCE-EVENT SIMILARITY}

In order to calculate the similarity between an instance and an event, we adopt a concept expansion strategy as in [3]. Our method is similar in spirit, however, we apply at instance level which is more accurate. The outline of our method is illustrated in Fig. 2 and it consists of four steps.

Step 1: Concept detection. We use the concept collection that proposed in [11] to cover a wide range of concept that can appear in realistic videos. This collection contains $\mathrm{C}=1183$ categories including 205 scene categories from the Places Database and 978 object categories from the ImageNet 2012. The concept detection part is done by using the provided pre-trained model ${ }^{1}$. To detect concept for the whole segment, we detect concept at sample frames and make the average aggregation.

Step 2: Event representation. We use standard natural language processing techniques to create the text-based event representation. At first, the event description is preprocessed by removing stop words and lemmatizing. It is then converted into a bag-of-words representation, where the dictionary is obtained from the English Wikipedia corpus. Tf-idf weighting scheme is also employed to put a higher weight on frequent as well as rare words.

Step 3: Concept-event similarity. To resolve the mismatch between words in the concept collection and event description, we adopt the concept expansion strategy [3]. For each concept category, we add the 10 most similar concepts obtained from word2vec model ${ }^{2}$ to expand this category. It is then represented by a bag-of-words vector with

\footnotetext{
${ }^{1}$ http://places.csail.mit.edu

${ }^{2}$ https://code.google.com/p/word2vec
}
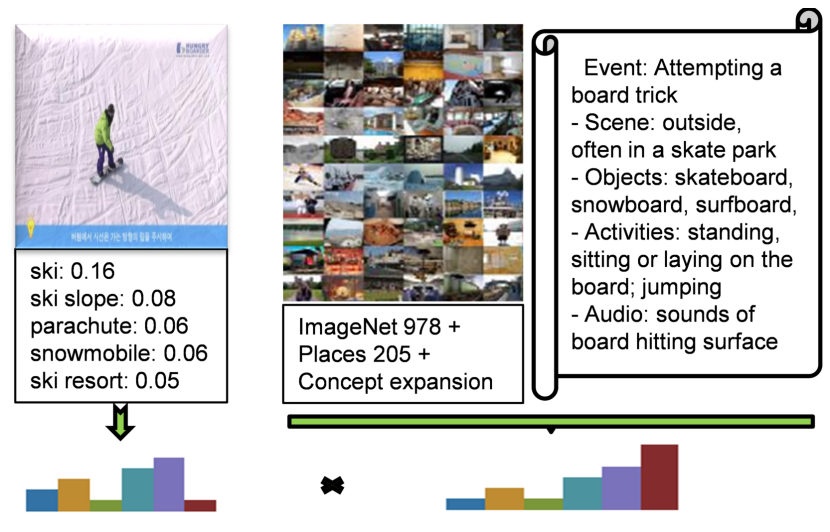

Figure 2: Outline of our method to calculate the instance-event similarity. Note that the concept expansion technique can bridge concept "ski" in the instance segment to the evidential description.

tf-idf weights. Based on this representation, we can calculate the cosine similarity $s_{c}^{e}$ between each concept category and the event description. Table 1 shows top five most relevant concepts for some events on the MED 2012 dataset.

Step 4: Instance-event similarity. Having obtained the concept score $x_{c}$ at each segment and the concept-event similarity as in Step 1 and Step 3, the instance-event similarity is calculated using the cosine similarity:

$$
S_{i}^{e}=\frac{\sum_{c=1}^{C} s_{c}^{e} x_{c}}{\sqrt{\sum_{c=1}^{C}\left(s_{c}^{e}\right)^{2}} \sqrt{\sum_{c=1}^{C}\left(x_{c}\right)^{2}}},
$$

\section{EVENT-DRIVEN MULTIPLE INSTANCE LEARNING}

Problem Formalization. Suppose we have $V$ training videos, and $I_{v}$ instances in video $v$. We can calculate the similarity $S_{i v}^{e}$ between an instance $i v$ to a particular event $e$ using Eq. (1). Suppose there is R level of relatedness from an instance to an event. We define two predict functions for positive and negative instances at level $r$ as follows.

$$
\begin{gathered}
P_{\text {pos }}\left(S_{i v}^{e}, r\right)=\left\{\begin{array}{ll}
1, & \text { if } \operatorname{Rank}\left(S_{i v}^{e}\right) \leq r \\
-1, & \text { otherwise }
\end{array},\right. \text { and } \\
P_{n e g}\left(S_{i v}^{e}, r\right)=\left\{\begin{array}{ll}
-1, & \text { if } \operatorname{Rank}\left(S_{i v}^{e}\right) \leq r \\
1, & \text { otherwise }
\end{array},\right.
\end{gathered}
$$

where $\operatorname{Rank}($.$) is the function to quantize a similarity into$ a related level. Note that smaller value of $r$ results a higher confidence in the predict functions. We now learn the parameters of the instance classifier jointly with the related level $r$ by optimizing the following objective function:

$$
\begin{array}{r}
\min _{\mathbf{w}, b, y, r} \frac{1}{2}\|\mathbf{w}\|^{2}+ \\
C_{f} \sum_{v=1}^{V} \sum_{i=1}^{I_{m}} L_{f}\left(y_{i v}, \mathbf{w}^{T} \mathbf{x}_{i v}+b\right) \\
+C_{p} \sum_{v=1}^{V} \sum_{i=1}^{I_{m}} L_{p}\left(y_{i v}, P\left(S_{i v}^{e}, r\right)\right) .
\end{array}
$$

$C_{f}$ and $C_{p}$ are cost parameters to control the influence of each loss function. Note that in the special case where 
Table 1: Top five concepts discovered by our system for the first 10 events in the MED 2012 dataset.

\begin{tabular}{|c|l|l|}
\hline Event ID & \multicolumn{1}{|c|}{ Event name } & \multicolumn{1}{c|}{ Concepts } \\
\hline E001 & Attempting a board trick & Ski, slide rule, ski resort, ski mask, ice skating rink \\
\hline E002 & Feeding an animal & Meat loaf, white shark, food court, pop bottle, cleaver \\
\hline E003 & Landing a fish & Anemone fish, pole, raft, sturgeon, boat deck \\
\hline E004 & Wedding ceremony & Groom, bridegroom, banquet hall, gown, altar \\
\hline E005 & Working on a woodworking project & Jigsaw puzzle, bamboo forest, carpenter's kit, thatch, wooden spoon \\
\hline E006 & Birthday party & Table lamp, lampshade, torch, candle, custard apple \\
\hline E007 & Changing a vehicle tire & Recreational vehicle, car wheel, amphibian, scooter, sports car \\
\hline E008 & Flash mob gathering & Monitor, chime, bell, whistle, ballroom \\
\hline E009 & Getting a vehicle unstuck & Recreational vehicle, amphibian, tank, car wheel, motor scooter \\
\hline E010 & Grooming an animal & Nail, bathtub, shower, fur coat, washbashin \\
\hline
\end{tabular}

$C_{p}=0$, the above formulation becomes a classic largemargin problem. $L_{f}($.$) and L_{p}($.$) are two loss functions$ that will be jointly minimized. The first loss function minimizes the loss due to the classification mismatch based on the instance feature. The second one minimizes the loss due to the prediction obtained from the prior knowledge. Intuitively, when the related level $r$ increases, the first loss will also tend to increase while the second loss will become smaller, and vice versa. $L_{f}($.$) and L_{p}($.$) can be any$ loss function. Throughout this paper, we use the standard hinge-loss function for $L_{f}():. L_{f}\left(y_{i v}, \mathbf{w}^{T} \mathbf{x}_{i v}+b\right)=$ $\max \left(0,1-y_{i v}\left(\mathbf{w}^{T} \mathbf{x}_{i v}+b\right)\right)$, and the $L_{p}($.$) function is defined$ so that it will penalize more on the high confident predictions: $L_{p}\left(y_{i v}, P\left(S_{i v}^{e}, r\right)\right)=\left\{\begin{array}{ll}S_{i v}^{e}, & \text { if } P\left(S_{i v}^{e}, r\right) \neq y_{i v} \\ 0, & \text { otherwise }\end{array}\right.$.

Optimization Procedure. The optimization problem in Eq. (4) is a mixed-integer program which is not convex. In order to solve this problem, we apply the alternating optimization strategy to search for a suboptimal solution:

1. Fix instance labels $y_{i v}$ and solve for $\mathbf{w}$ and $b$. By fixing $y_{i v}$, the optimization problem becomes a classic SVM: $\min _{\mathbf{w}, b} \frac{1}{2}\|\mathbf{w}\|^{2}+C_{f} \sum_{v=1}^{V} \sum_{i=1}^{I_{v}} L_{f}\left(y_{i v}, \mathbf{w}^{T} \mathbf{x}_{i v}+b\right)$. Thus it can be solved using a regular SVM solver.

2. Fix $\mathbf{w}$ and $b$, solve for $r$ and update $y_{i v}$. The problem now becomes: $\min _{y, r} C_{f} \sum_{v=1}^{V} \sum_{i=1}^{I_{v}} L_{f}\left(y_{i v}, \mathbf{w}^{T} \mathbf{x}_{i v}+b\right)$ $+C_{p} \sum_{v=1}^{V} \sum_{i=1}^{I_{v}} L_{p}\left(y_{i v}, P\left(S_{i v}^{e}, r\right)\right)$. We propose a greedy strategy to solve for this problem. At first, we iterate through all level of relatedness to search for the optimal $r$ by finding the minimum total loss when updating $y_{i v}$ using Eq. $(2,3)$. Because the most positive and negative instances will be selected first, there will be a higher possibility to correct mismatched labels that were learned in the previous step. Lastly we update instance labels using Eq. $(2,3)$ with the optimal $r$.

Because this is not a convex optimization problem, the initialized values of $y_{i v}$ should be carefully selected. To this end, we use the same initilization method as in [1,5], where instance labels are same with its "bag" (video) label.

It is also worth noted that the optimization framework only keeps updating the instance labels while the instance features are unchanged. Thus it is a good practice to use the pre-computed kernel technique for optimizing $\mathbf{w}$ and $b$. In fact, although our method is more complex, it only takes around 5 minutes for training one model, compared to 40 minutes that was reported in [5].

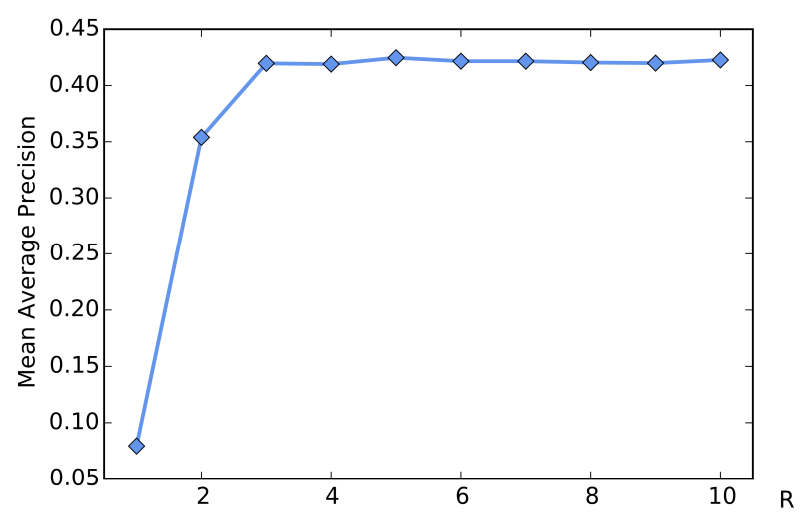

Figure 3: Optimal number of related levels.

\section{EXPERIMENTS}

Dataset. To evaluate our proposed method, we conducted experiments on the large scale TRECVID MED 2012 dataset $^{3}$. This dataset provides the definition for 25 complex events. The first ten event names are listed in Table 1. We follow the setting by [5] to divide this video collection into training and testing parts. These parts contain 3,878 and 1,938 videos respectively.

Experimental setup. At first, original videos are scaled down to $320 \times 240$ with keeping the aspect ratio. Key frames are sampled at every 2 seconds from the resized video. The segment length is set to 8 seconds as suggested in [8]. To extract feature for each segment, we use the Improved Dense Trajectories feature proposed by Wang and Schmid [9]. Motion Boundary Histogram (MBH) is used to represent extracted trajectories because it can handle camera motion, which is prevalent in realistic videos. For learning, we use our framework jointly with the linear SVM. The cost parameters $C_{f}$ and $C_{p}$ are selected by cross-validation in the range of $\{0.1,1,10,100\}$. At the testing step, video-level score is obtained by averaging over all instance scores. Finally we use the standard evaluation metric on MED, Mean Average Precision (mAP), to report the performance.

Baseline methods. To compare our methods with following baselines: miSVM, MISVM [1], VideoBOW and pSVM [5]. At first, because our method is based on the Multiple Instance Learning (MIL) framework, we evaluate two MIL solutions: miSVM and MISVM that were proposed by the

\footnotetext{
$\overline{{ }^{3} \text { http://www.nist.gov/itl/iad } / \mathrm{mig} / \operatorname{med} 12 . \mathrm{cfm}}$
} 


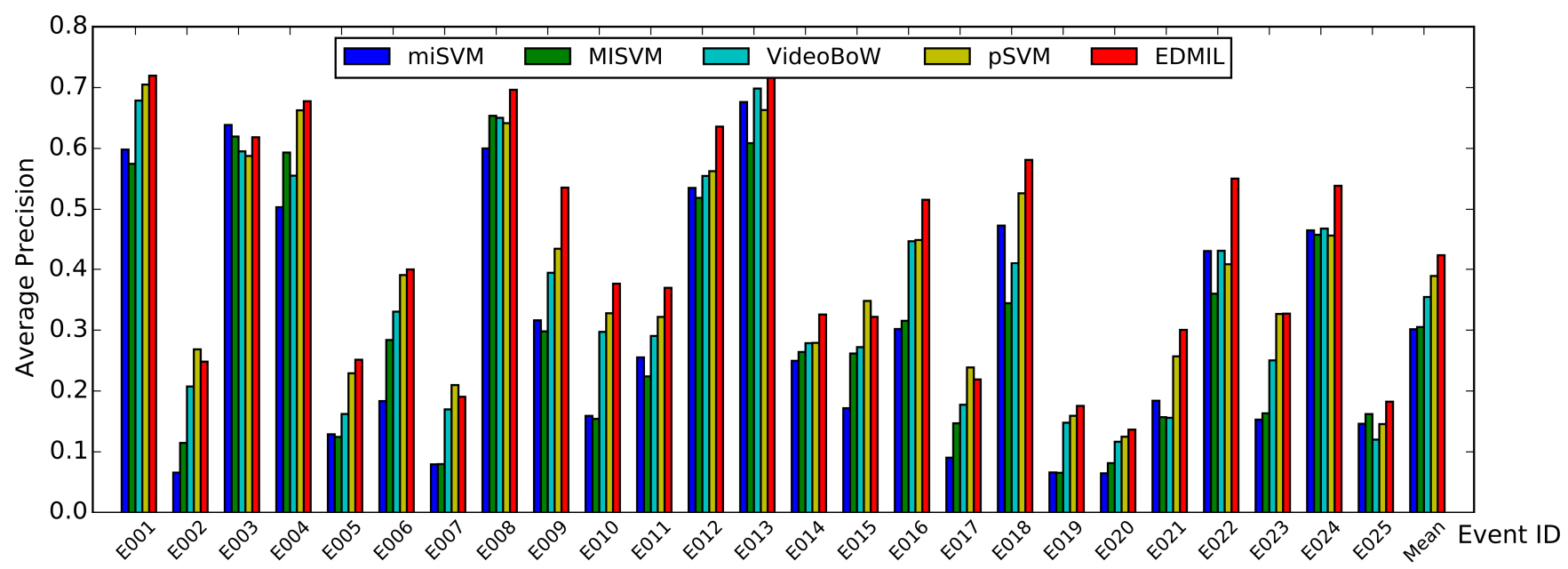

Figure 4: Evaluation results of 25 events in the TRECVID MED 2012 dataset. The mean APs are 0.3015 (miSVM), 0.3051 (MISVM), 0.3544 (VideoBOW), 0.3890 (pSVM) and 0.4246 (Ours).

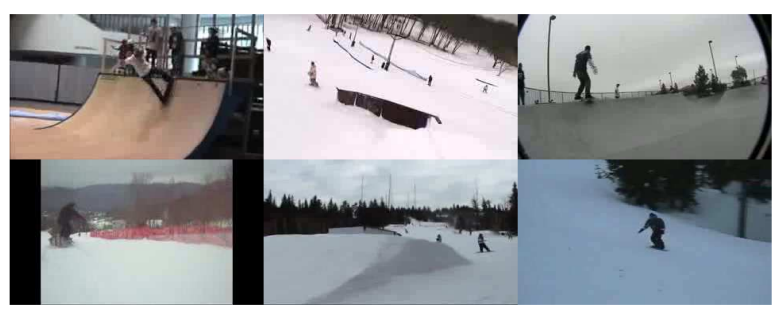

Figure 5: The top 6 key evidences detected by our system for the event "Attempting board trick". The dominance of ski-related instances is reasonable.

authors in [1]. The VideoBOW method is the standard approach where local features are aggregated from the whole video. We also compare our method with the recently proposed pSVM which was adopted in [5] for TRECVID MED. For all the baseline methods, except VideoBOW, we utilize the codes provided by the authors to test with our features.

Experimental results. At first, we conduct experiments to find the optimal value of $R$. We select $R$ in the range from 1 to 10. The overall performance is shown in Fig. 3. We obtain the peak performance with $\mathrm{R}$ around 5. Small values of $R$ tend to get low performances. This indicates that the prediction of prior knowledge is not always good, and learning jointly with instance features is necessary. The performance becomes saturated when $\mathrm{R}>5$. Therefore, we fix the value of $\mathrm{R}$ to 5 for further experiments.

The performance of each baseline method as well as our method (EDMIL) are shown in Fig. 4. Our method significantly outperforms other baselines. For the best baseline, our method relatively outperforms by $10 \%$. Our instancebased classifier can also provide key evidences for event detection. Example of key evidences detected by our system can be seen in Fig. 5 .

\section{CONCLUSIONS}

We propose a new method to detect event in videos from its key evidences. Our method differs from others in that we utilize the evidential description provided for each event.
Given this supportive information, we search for key evidences by jointly optimizing with instance feature in a variant of multiple instance learning framework. As a result, we obtained a superior event detection performance.

\section{REFERENCES}

[1] S. Andrews, I. Tsochantaridis, and T. Hofmann. Support vector machines for multiple-instance learning. In NIPS, pages 561-568, 2002.

[2] S. Bhattacharya, F. X. Yu, and S.-F. Chang. Minimally needed evidence for complex event recognition in unconstrained videos. In ICMR, 2014.

[3] J. Chen, Y. Cui, G. Ye, D. Liu, and S.-F. Chang. Event-driven semantic concept discovery by exploiting weakly tagged internet images. In ICMR, 2014.

[4] K.-T. Lai, D. Liu, M.-S. Chen, and S.-F. Chang. Recognizing complex events in videos by learning key static-dynamic evidences. In ECCV. 2014.

[5] K.-T. Lai, F. X. Yu, M.-S. Chen, and S.-F. Chang. Video event detection by inferring temporal instance labels. In CVPR, pages 2251-2258. IEEE, 2014.

[6] D. Oneata, J. Verbeek, and C. Schmid. Action and event recognition with fisher vectors on a compact feature set. In ICCV. IEEE, 2013.

[7] K. Tang, L. Fei-Fei, and D. Koller. Learning latent temporal structure for complex event detection. In CVPR, pages 1250-1257. IEEE, 2012.

[8] A. Vahdat, K. Cannons, G. Mori, S. Oh, and I. Kim. Compositional models for video event detection: A multiple kernel learning latent variable approach. In ICCV, pages 1185-1192. IEEE, 2013.

[9] H. Wang and C. Schmid. Action recognition with improved trajectories. In ICCV. IEEE, 2013.

[10] S. Wu, S. Bondugula, F. Luisier, X. Zhuang, and P. Natarajan. Zero-shot event detection using multi-modal fusion of weakly supervised concepts. In CVPR, pages 2665-2672. IEEE, 2014.

[11] B. Zhou, A. Lapedriza, J. Xiao, A. Torralba, and A. Oliva. Learning Deep Features for Scene Recognition using Places Database. NIPS, 2014. 